\title{
An automatically contamination-avoiding technique for intracorporeal esophagojejunostomy using a transorally inserted anvil during laparoscopic total gastrectomy for gastric cancer
}

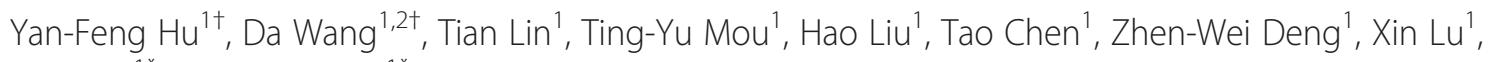
Jiang $\mathrm{Yu}^{1 *}$ and Guo-Xin $\mathrm{Li}^{1^{*}}$

\begin{abstract}
Background: Intracorporeal Roux-en-Y esophagojejunostomy during laparoscopic total gastrectomy for gastric cancer remains a challenging manipulation due to the uncontrolled direction of the jejunal side or unintended embedded tissues, although several methods have been introduced. In this study, we simplified the procedure based on a surgical string fixing technique using a transorally inserted anvil (OrVil ${ }^{\mathrm{TM}}$; Covidien Ltd., Mansfield, MA, USA).

Methods: From March 2012 to September 2013, 14 consecutive patients underwent simplified intracorporeal Roux-en-Y esophagojejunostomy using OrVil ${ }^{\mathrm{TM}}$ during laparoscopic total gastrectomy for gastric cancer at our hospital. Clinicopathologic characteristics and surgical outcomes of these patients were retrospectively analyzed.

Results: All of the procedures were successful completed with no complication or conversion to open surgery. The mean overall operative time was $193.8 \pm 41.8 \mathrm{~min}$, whereas the mean reconstruction time was $32.6 \pm 4.6 \mathrm{~min}$. The mean estimated blood loss was $105.7 \pm 65.4 \mathrm{ml}$. The mean diameter of anastomosis measured by upper gastrointestinal contrast X-ray test at 1 month after operation was $2.3 \mathrm{~cm}$. During a median follow-up period of 12 months, neither local recurrence nor anastomosis-related morbidity was observed.
\end{abstract}

Conclusions: Our preliminary results suggested that this automatically contamination-avoiding technique based on a surgical-string-fixing strategy using OrVil ${ }^{\text {TM }}$ during laparoscopic total gastrectomy for gastric cancer might be feasible and safe and provide a simple solution for intracorporeal Roux-en-Y esophagojejunostomy.

Keywords: Gastric cancer, Laparoscopy, Esophagojejunostomy, Gastrectomy

\section{Background}

Extracorporeal Roux-en-Y esophagojejunostomy during laparoscopy-assisted total gastrectomy for gastric cancer is one of the most challenging manipulations even in experienced hands. To overcome the surgical difficulty and maximize the clinical benefits from minimally invasive surgery in the management of patients with proximal gastric cancer, intracorporeal esophagojejunostomy, including either circular or linear anastomosis, has been introduced

\footnotetext{
*Correspondence: balbc@163.com; gzliguoxin@163.com

${ }^{\dagger}$ Equal contributors

'Department of General Surgery, Nanfang Hospital, Southern Medical University, No. 1838 The North Guangzhou Avenue, Guangzhou 510515 Guangdong, China

Full list of author information is available at the end of the article
}

recently [1-4]. Among those methods, circular anastomosis following transabdominal insertion of an anvil with purse-string suture is the most frequently used pattern $[1,4]$. However, the difficulties in terms of laparoscopically creating a purse-string suture and fixing the anvil at the esophageal stump always exist, increasing the potential risk of complications and prolonging the operation time.

To avoid these difficulties mentioned above, a transorally inserted anvil (OrVil ${ }^{\mathrm{Tw}}$; Covidien Ltd., Mansfield, MA, USA) device has been developed in recent years [5-7]. Previous reports $[6,8]$ have shown its technical feasibility and potential advantages; however, the course of approximation between the anvil and center rod does not always proceed safely by intracorporeal laparoscopic view due to






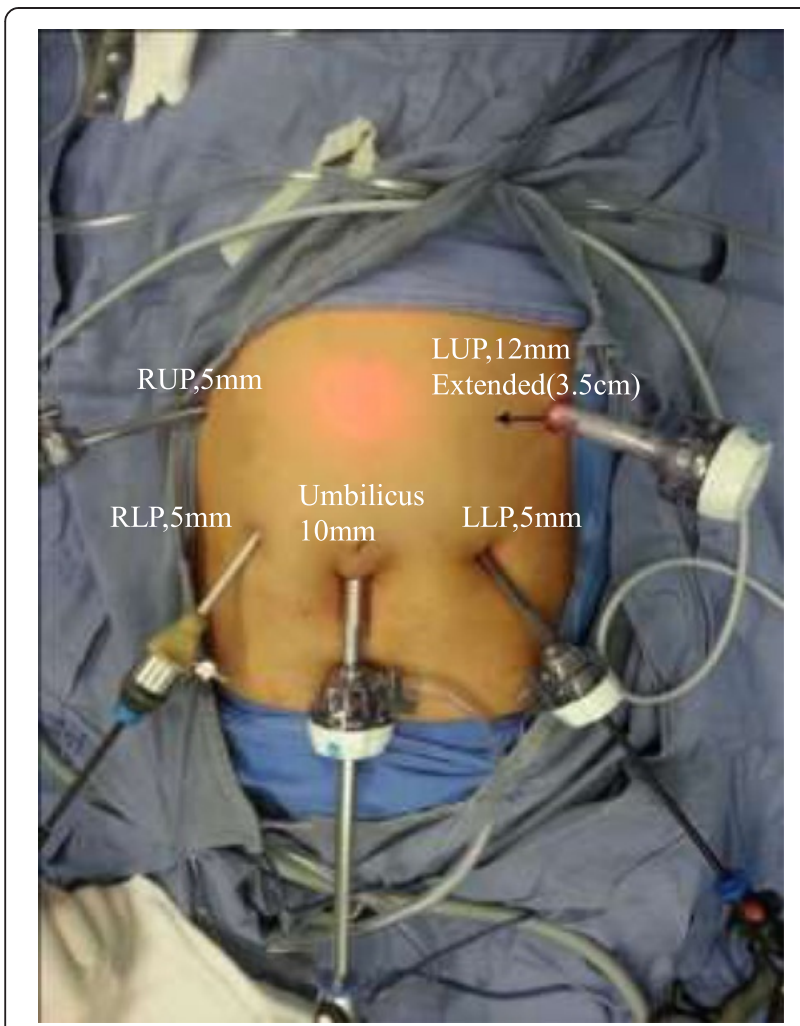

Figure 1 Placement of the trocars. The left upper port site will be extended transversely to an incision length of 3 to $4 \mathrm{~cm}$ for extraction of the specimen and insertion of the circular stapler before anastomosis. RUP, right upper port; LUP, left upper port; RLP, right lower port; LLP, left lower port. the uncontrolled direction of the jejunal side or unintended embedded tissues $[7,8]$. Thus, we attempted to simplify this technique by adopting several tips with inexpensive and available devices and evaluated its surgical safety in the present study.

\section{Methods \\ Patients}

Between March 2012 and September 2013, 14 consecutive patients with proximal gastric adenocarcinoma underwent simplified intracorporeal Roux-en-Y esophagojejunostomy using a transorally inserted anvil system (OrVil ${ }^{\mathrm{m}}$; Covidien, Mansfield, MA, USA) during laparoscopic total gastrectomy at our hospital. These patients followed a stepwise postoperative management protocol for diet resume from water to other liquids to semifluids to normal food when the patient can tolerate the diet satisfactorily and is free from anastomotic complication as early as possible after surgery and received upper gastrointestinal contrast X-ray check of esophagojejunostomy at postoperative 1 month. Clinicopathologic characteristics and surgical outcomes of these patients based on a prospectively maintained database [9] were then retrospectively analyzed. The present study was approved by the Ethics Committee of Nanfang Hospital (No. 2013087A).

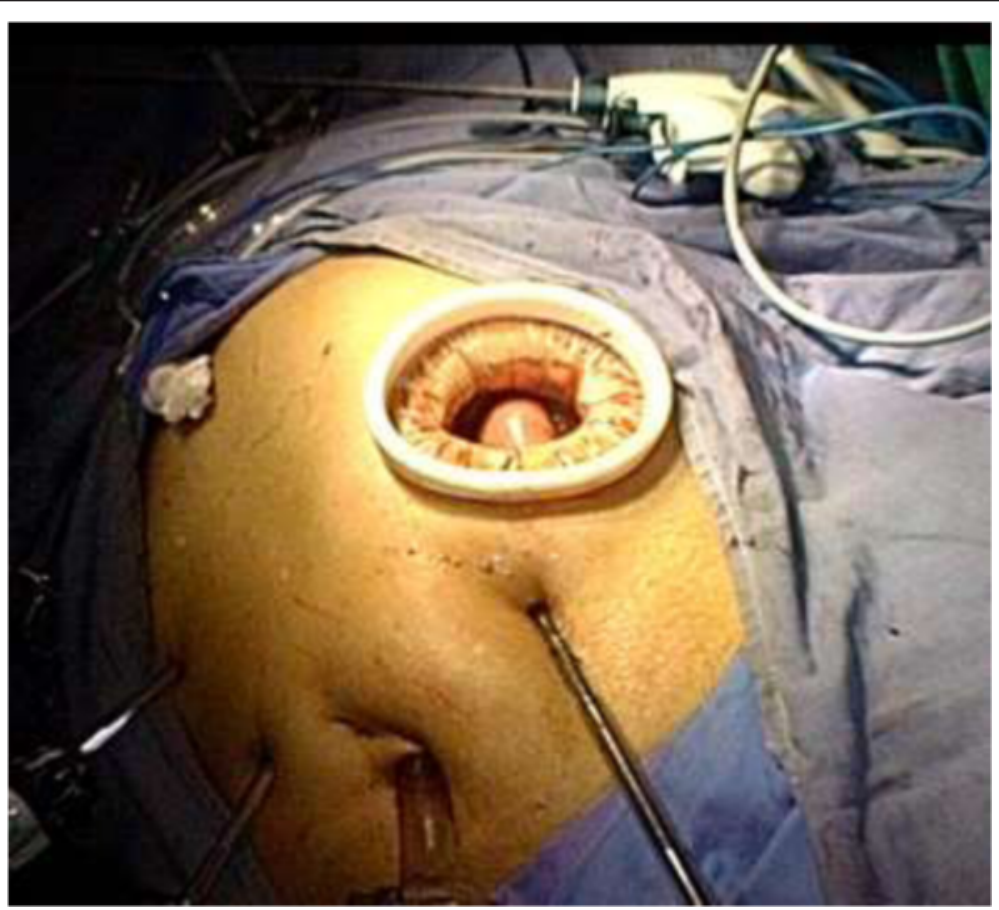

Figure 2 Minilaparotomy at the left upper quadrant for specimen retrieval and stapler insertion. 


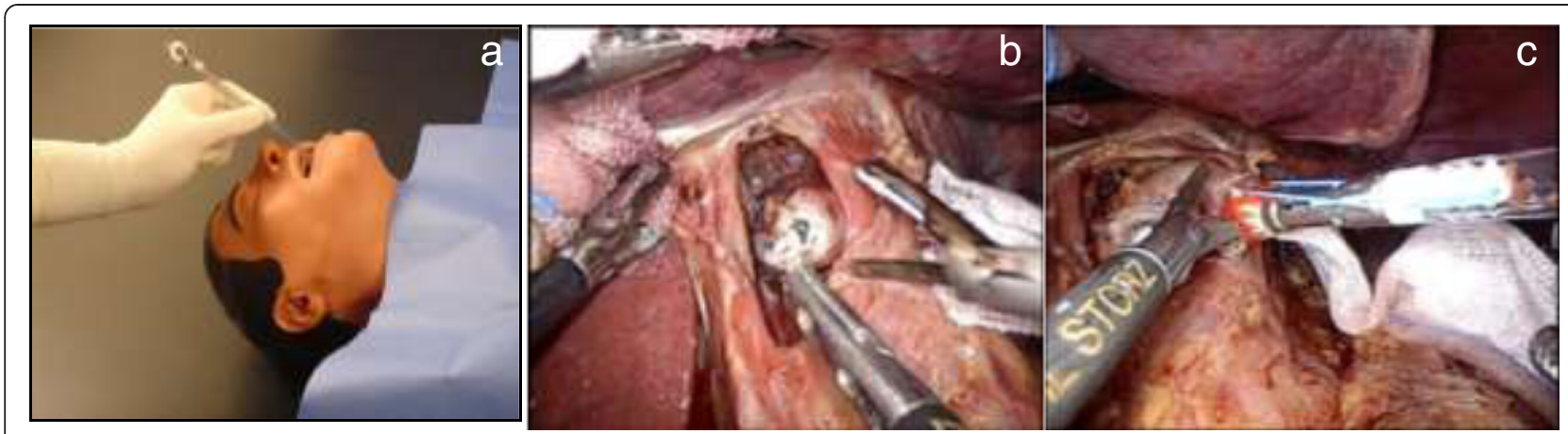

Figure 3 Placement of the anvil head. (a) The tube of the anvil head was inserted transorally (picture downloaded from the website of Covidien). (b) A small hole was made at the esophageal stump. (c) The thread was cut after fixation of the anvil head.

\section{Procedure before anastomosis}

Under general anesthesia, the patient was placed in the supine position with legs slightly apart (relaxed dorsal lithotomy position). The operator and assistant stood on the patient's left and right side, respectively, and the camera holder stood between the legs of the patient. Trocar placement is shown in Figure 1.

To obtain better exposure of the operative field, the falciform ligament and liver were suspended to the abdominal wall using Hyung's strategy [4]. After dissection of the

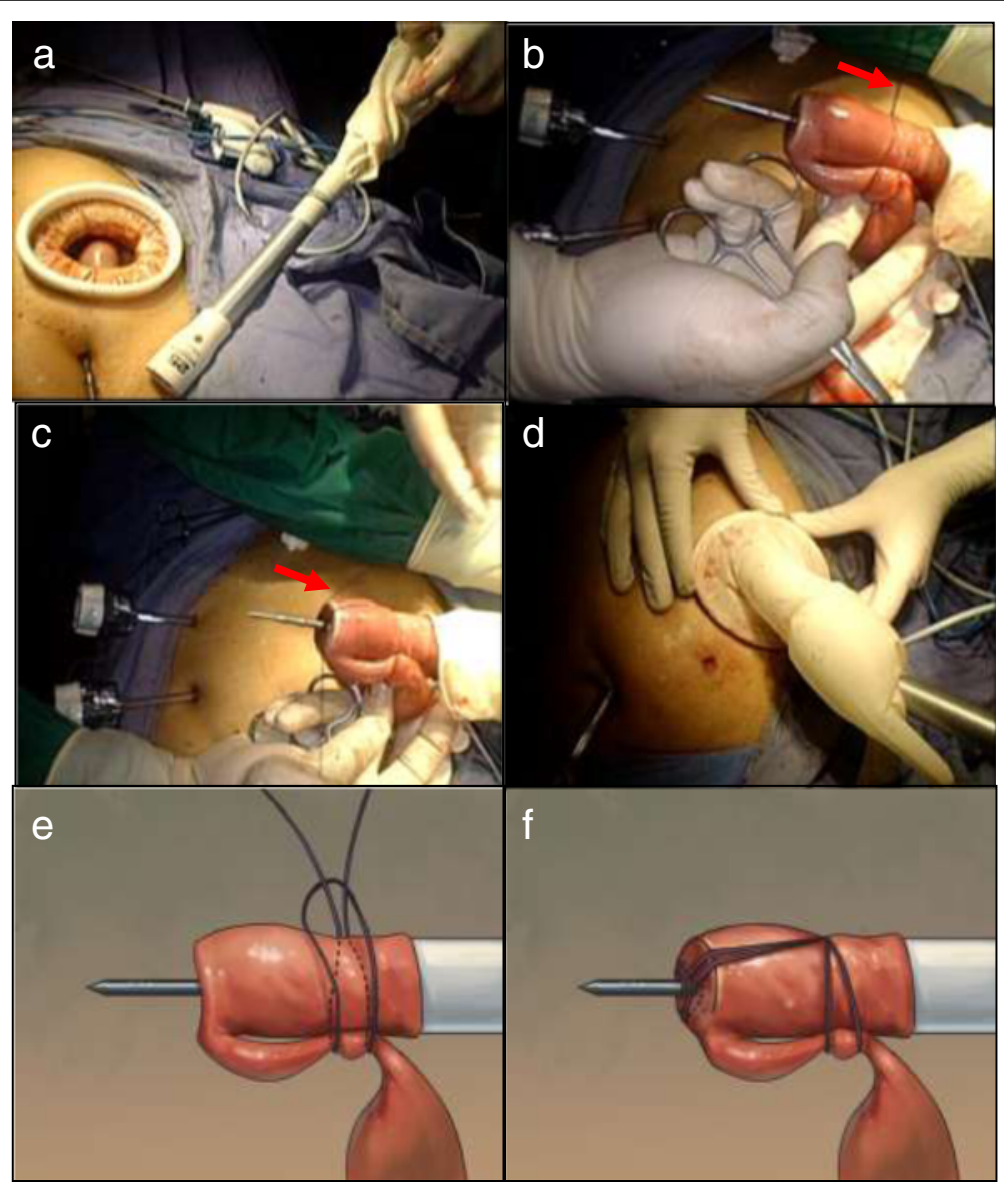

Figure 4 A self-made single-site access system. (a) The circular stapler passed through the glove. (b) The stapler, jejunal stump, and loop were fixed by the silk string. (c) A slipknot was made to fix the shaft. (d) Establishment of the pneumoperitoneum by the self-made single-site access system. (e) Schematic of making a slipknot using a silk string. (f) Schematic of making a surgical knot to the center rod. Red arrow points to the silk suture. The knot was released automatically during firing of the stapler without additional cutting. 
regional lymph nodes, the duodenal bulb was transected using a linear stapler laparoscopically, followed by transection of the distal esophagus with a proper distance from the lesion using a linear stapler with flexible articulation. Next, the entire specimen was removed through a 3- to $4-\mathrm{cm}$ minilaparotomy incision extending to the left upper port site, where a wound protector (Beijing HangTian $\mathrm{KaDi}$ Technology R \& D Institute, Beijing, China) was used (Figure 2).

\section{Anastomosis}

\section{Insertion of the anvil transorally}

A small hole was created at one angle of the esophageal stump laparoscopically. Concurrently, an anesthetist inserted the tube of the anvil $\left(\mathrm{OrVil}^{\mathrm{m}} \mathrm{m}\right)$ transorally until the tip of the tube passed through the small hole at the esophageal stump (Figure 3a, b). A laparoscopic grasper was then used to slowly drag the tube until the anvil rod came into view.

The tube was lightly secured to fix the anvil at the proper position. The thread connecting the tube and anvil was then released (Figure 3c). The tube was removed to expose the rod of the anvil sufficiently. At that time, the insertion of the anvil was completed.

\section{Modification and tips of the intracorporeal esophagojejunostomy}

The jejunum was transected $15 \mathrm{~cm}$ away from the Treitz's ligament using a linear stapler laparoscopically. A surgical glove, a minilaparotomy wound protector, and the shaft of the stapler were integrated as a selfmade single-site access system (Figure 4). The stapler was then positioned within the jejunal loop from the distal jejunal stump. Both the jejunal end and loop were anchored on the main unit with a silk string to create a slipknot to fix the center rod to prevent separation and avulsion (Figure 4).

\section{Approximation between the anvil and center rod}

The anvil and circular stapler were connected, and anastomosis was performed under laparoscopic view directly

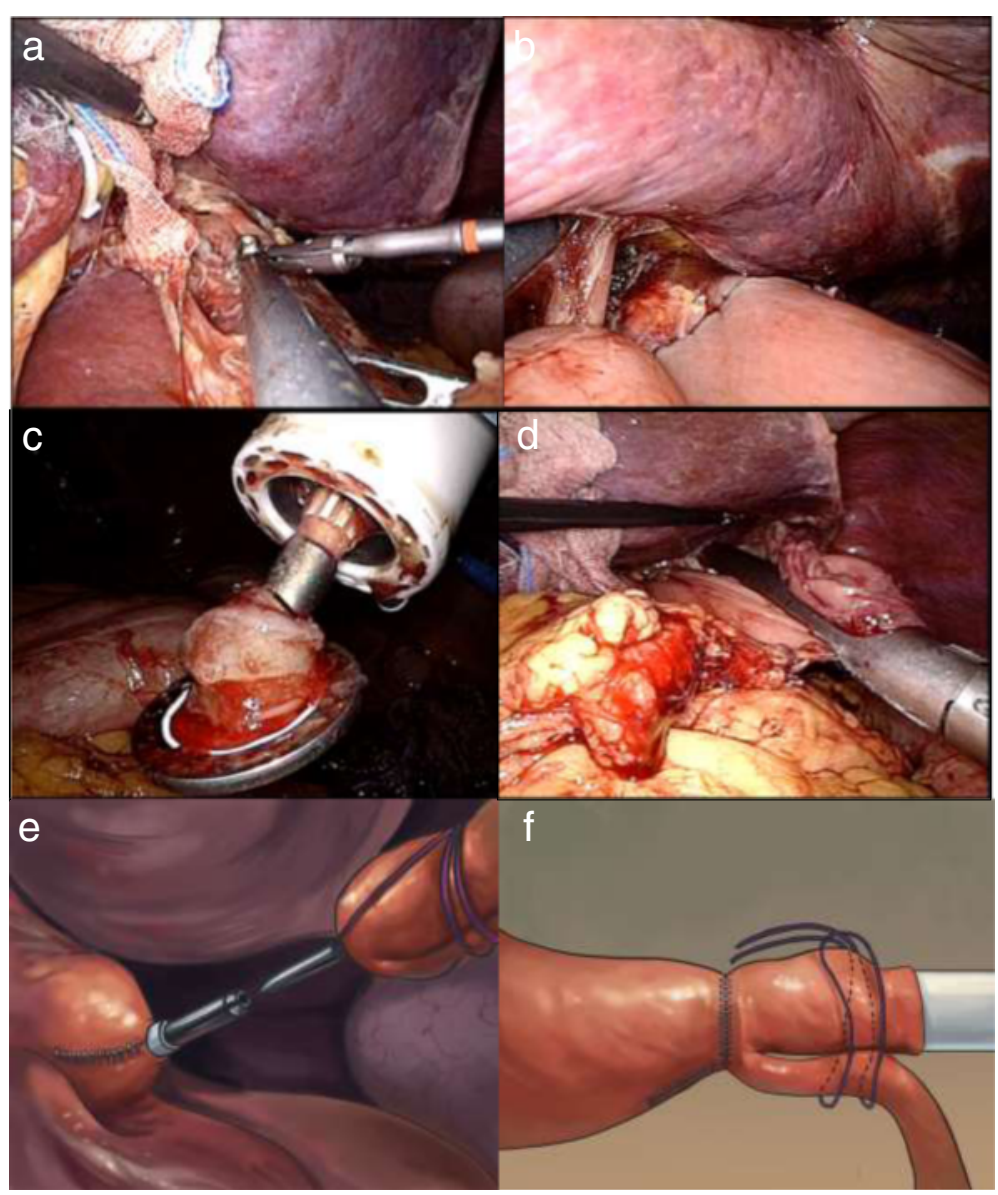

Figure 5 Intracorporeal anastomotic technique using a circular stapler. (a) Connection of the shaft and anvil. (b) Approximation of the shaft and anvil. The knot was released automatically during firing of the stapler. (c) The stapler was carefully removed. (d) The jejunal stump was closed using a linear stapler. (e) Schematic of approximation between the anvil and center rod. (f) Schematic of automatically removing an anchoring string during firing. 
(Figure 5). Moderation by rotating the stapler was easily performed to make a fine alignment, precisely controlling the direction of the jejunal side with little concern about tearing embedded tissues. After confirming that the bilateral crura of the diaphragm were not embedded in the stapler (the anastomotic stoma was satisfactory), the stapler was fired and loosened. Simultaneously, an anchoring string on the small bowel and the circular stapler were removed automatically without additional cutting. The circular stapler was removed gently from the anastomotic bowel, and the quality of the anastomosis was checked by identifying the donuts on the stapler. The jejunal stump was closed 2 to $3 \mathrm{~cm}$ away from the esophagojejunal site using a linear stapler. After side-to-side jejunojejunostomy was performed by linear stapler laparoscopically, the continuity of the digestive tract was completed.

\section{Statistical analysis}

Demographic, operative, and clinicopathologic data of the patients are expressed as mean \pm standard deviation or median (range) if they are continuous variables.

\section{Table 1 Clinicopathologic characteristics and operative} results of the patients

\begin{tabular}{|c|c|}
\hline Variable & Value \\
\hline \multicolumn{2}{|l|}{ Demography } \\
\hline Male/female, $n$ & $10 / 4$ \\
\hline Age, years & $59.0 \pm 12.1$ \\
\hline Body mass index, $\mathrm{kg} / \mathrm{m}^{2}$ & $23.4 \pm 2.2$ \\
\hline \multicolumn{2}{|l|}{ Operation } \\
\hline Overall operation time, min & $193.8 \pm 41$. \\
\hline Overall reconstruction time, min & $32.6 \pm 4.6$ \\
\hline Time for anvil placement, min & $8.4 \pm 4.0$ \\
\hline Time for esophagojejunostomy, min & $23.8 \pm 5.2$ \\
\hline Estimated blood loss, ml & $105.7 \pm 65$ \\
\hline \multicolumn{2}{|l|}{ Pathology } \\
\hline Stage ${ }^{a} \mid / I I / I I I, n$ & $1 / 3 / 10$ \\
\hline Proximal margin, $\mathrm{cm}$ & $3.6 \pm 1.7$ \\
\hline No. of retrieved lymph nodes & $33.9 \pm 18.1$ \\
\hline \multicolumn{2}{|l|}{ Immediate postoperative course } \\
\hline First flatus, mean, days & $3.3 \pm 0.7$ \\
\hline Hospital duration, mean, days & $8.7 \pm 3.2$ \\
\hline Mortality, $n$ & 0 \\
\hline Short-term anastomosis-related complications, $n$ & 0 \\
\hline Esophagojejunostomy diameter, cm & $2.3 \pm 0.3$ \\
\hline \multicolumn{2}{|l|}{ Follow-up } \\
\hline Follow-up period (median, range), months & 12 (6 to 24$)$ \\
\hline Long-term complications, $n$ & 0 \\
\hline
\end{tabular}

Values are expressed as mean \pm standard deviation unless otherwise indicated.

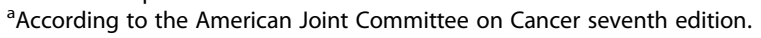

\section{Results}

The clinicopathologic features and operative results of the patients are shown in Table 1. The mean overall operation time was $193.8 \pm 41.8 \mathrm{~min}$. The mean reconstruction time was $32.6 \pm 4.6 \mathrm{~min}$. No intraoperative complication was observed. The mean first flatus time and hospital duration were $3.29 \pm 0.73$ and $8.7 \pm 3.2$ days, respectively. The mean diameter of anastomosis measured by upper gastrointestinal contrast X-ray test at 1 month after operation was $2.3 \mathrm{~cm}$ (Figure 6). During a median follow-up period of 12 months, neither local recurrence nor anastomosisrelated morbidity was observed.

\section{Discussion}

Laparoscopic total gastrectomy becomes significantly challenging when the reconstruction procedure involves intracorporeal esophagojejunostomy because it is difficult to perform a proper purse-string suture at the esophageal stump and insert an anvil into the esophageal lumen [4]. Even more troublesome is applying the stapling device appropriately under limited laparoscopic views, particularly in obese patients [7]. Some reports have introduced solutions to improve the procedure, but

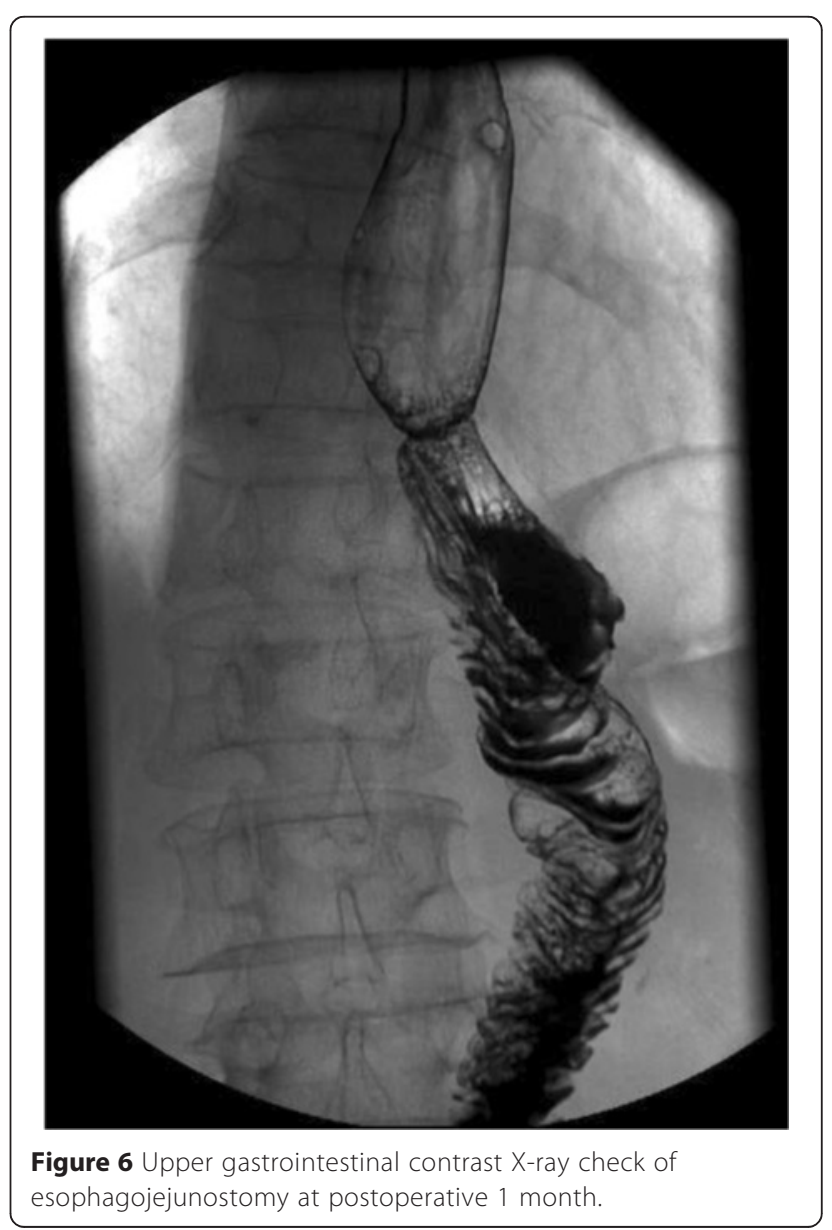


problems remain such as the requirement for special expensive instruments that are not always available or additional skills $[4,10,11]$. A linear stapler has been chosen by some surgeons instead of a circular one to perform esophagojejunostomy laparoscopically that unsatisfactorily overcomes the limitation of requiring a certain esophageal length and an intracorporeal hand-suture [3].

To solve these problems and overcome the limitations, we describe herein a simplified technique for intracorporeal esophagojejunostomy using a circular stapler. No special technique is required, and the simplified procedure can be easily performed. Its safety and feasibility has been suggested in a series of 14 patients, of whom none suffered postoperative anastomosis-related complications.

Transoral insertion of an anvil $\left.\left(\mathrm{OrVil}^{\mathrm{m}}\right)^{\mathrm{m}}\right)$ provides a solution for secure anvil placement in the esophagus. With an anesthesiologist's help, pharyngeal or esophageal injury can be effectively avoided. Our method to manipulate the process is to open a small incision at the alternative edge of the esophageal stump after dividing the esophagus horizontally using a linear stapler and securing the anvil with a pair of grasping forceps after introducing the anvil tube into view. Thus, the anvil is guaranteed to be placed at an appropriate position without tearing the esophageal stump facilitating subsequent operations.

With similar methods in the recently published literature [7], problems such as distortion of the Roux limb or mesenterium and slipping of the esophagojejunal anastomotic site into the low mediastinum have not yet met ideal solutions. As presented above, fixation of the circular stapler and glove was followed by fixation of the jejunal loop and glove, causing the stapler-glove-jejunal loop to integrate and form a self-made single-site access system that prevents slippage of the jejunal loop out of the circular stapler during the intracorporeal procedure. Additionally, flexible mobilization is enabled to make a fine alignment, ensuring optimal functioning of the reconstructive digestive tract. The small bowel was more easily held backward to prevent it from becoming trapped between the anvil and circular stapler. The sealed space, established by turning over the glove edge to seal off the wound protector, allowed for a clearer visual field for the laparoscopic operation. Thus, tissue injury caused by excessive traction or slight errors can be minimized. Moreover, the anchoring suture could be cut along with the donut during the scalper firing and separated automatically without additional cutting when the main unit was pulled back. Thus, the simplified technique does not significantly create excessive steps and prolong the overall operative time. To prevent possible anastomotic stenosis, selection of the proper anvil size of circular stapler should be considered carefully, which usually depends on both the diameter of esophageal and jejunal stump. A diameter of $25-\mathrm{mm}$ anvil is the most common choice at our institution for Chinese patients, while a relatively larger size would be applied casually. Meanwhile, intraoperative examination and postoperative early oral intake was also recommended [12]. In our daily clinical practice, generally, a stepwise management protocol for diet resume from water to other liquids to semi-fluids to normal food should be carried out when the patient can tolerate the diet satisfactorily and is free from anastomotic complication as early as possible after surgery. Theoretically, adding the solid food appropriately in terms of volume and frequency might be helpful to dilate the anastomotic site. Besides, routine upper gastrointestinal contrast X-ray test at postoperative 1 month is recommended for early detection of stricture.

\section{Conclusions}

Our initial findings show that this automatically contamination-avoiding technique based on a surgicalstring-fixing strategy is technically feasible and could provide a simple solution for intracorporeal Roux-en-Y esophagojejunostomy. However, a perspective study is needed to evaluate the surgical safety of this technique.

\section{Competing interests}

The authors declare that they have no competing interests.

\section{Authors' contributions}

YFH and DW contributed equally to this work and should be considered co-first authors. DW and YFH wrote the paper. YFH, DW, TL, TYM, HL, CT, $Z W D$, and $X L$ collected and analyzed the data. JY and GXL designed the study and reviewed the article. All authors read and approved the final manuscript.

\section{Acknowledgements}

This work was supported by the Research Fund of Public welfare in Health Industry (No. 201402015), the Major Program of Science and Technology Program of Guangzhou (No. 201300000087), the National Key Technology R \& D Program (no. 2013BAI05B00), and the Development Center for Medical Science and Technology, Ministry of Health of China (no. W2011WAI44), and the Key Clinical Specialty Discipline Construction Program.

\section{Author details}

${ }^{1}$ Department of General Surgery, Nanfang Hospital, Southern Medical University, No. 1838 The North Guangzhou Avenue, Guangzhou 510515, Guangdong, China. ${ }^{2}$ The Key Laboratory of Cancer Prevention and Intervention, China National Ministry of Education, Department of Surgical Oncology, The Second Affiliated Hospital of Zhejiang University School of Medicine, 88 Jie-Fang Road, Hangzhou 310009, Zhejiang Province, China.

Received: 5 November 2014 Accepted: 26 March 2015

Published online: 19 April 2015

\section{References}

1. Kinoshita T, Oshiro T, Ito K, Shibasaki H, Okazumi S, Katoh R. Intracorporeal circular-stapled esophagojejunostomy using hand-sewn purse-string suture after laparoscopic total gastrectomy. Surg Endosc. 2010;24:2908-12.

2. Jeong O, Park YK. Intracorporeal circular stapling esophagojejunostomy using the transorally inserted anvil (OrVil ${ }^{\mathrm{TM}}$ ) after laparoscopic total gastrectomy. Surg Endosc. 2009;23:2624-30.

3. Nagai E, Ohuchida K, Nakata K, Miyasaka Y, Maeyama R, Toma H, et al. Feasibility and safety of intracorporeal esophagojejunostomy after laparoscopic total gastrectomy: inverted T-shaped anastomosis using linear staplers. Surgery. 2013;153:732-8. 
4. Kim HI, Cho I, Jang DS, Hyung WJ. Intracorporeal esophagojejunostomy using a circular stapler with a new purse-string suture technique during laparoscopic total gastrectomy. J Am Coll Surg. 2013;216:e11-6.

5. Kunisaki C, Makino H, Oshima T, Fujji S, Kimura J, Takagawa R, et al. Application of the transorally inserted anvil (OrVi $\mathrm{I}^{\mathrm{T}}$ ) after laparoscopy-assisted total gastrectomy. Surg Endosc. 2011;25:1300-5.

6. Mou T, Hu Y, Yu J, Wang Y, Zhao L, Li G. Clinical value of transorally inserted anvil in esophagojejnnostomy after laparoseopic total gastrectomy for gastric cancer. Chin J Dig Surg. 2012;11:211-4.

7. Chong-Wei K, Dan-Lei C, Dan D. A modified technique for esophagojejunostomy or esophagogastrostomy after laparoscopic gastrectomy. Surg Laparosc Endosc Percutan Tech. 2013;23:e109-15.

8. Liao GQ, Ou XW, Liu SQ, Zhang SR, Huang W. Laparoscopy-assisted total gastrectomy with trans-orally inserted anvil (OrVil): a single institution experience. World I Gastroenterol. 2013;19:755-60.

9. Hu YF, Yu J, Zhang C, Wang YN, Cheng X, Huang F, et al. Development and implementation of a clinical data mining system for gastric cancer surgery. Zhonghua Wei Chang Wai Ke Za Zhi. 2010;13:510-5.

10. Facy O, De Blasi V, Goergen M, Arru L, De Magistris L, Azagra JS. Laparoscopic gastrointestinal anastomoses using knotless barbed sutures are safe and reproducible: a single-center experience with 201 patients. Surg Endosc. 2013;27:3841-5.

11. Yoshikawa T, Hayashi T, Aoyama T, Cho H, Fujikawa H, Shirai J, et al, Laparoscopic esophagojejunostomy using the EndoStitch and a circular stapler under a direct view created by the ENDOCAMELEON. Gastric Cancer. 2013;16:609-14.

12. Inagake M, Yamane T, Kitao Y, Okuzumi J, Kuwata K, Yamaguchi T, et al. Balloon dilatation for anastomotic stricture after upper gastro-intestinal surgery. World J Surg. 1992;16:541-4.

\section{Submit your next manuscript to BioMed Central and take full advantage of:}

- Convenient online submission

- Thorough peer review

- No space constraints or color figure charges

- Immediate publication on acceptance

- Inclusion in PubMed, CAS, Scopus and Google Scholar

- Research which is freely available for redistribution 